\title{
Variabilidad y variantes de la enfermedad de Alzheimer
}

\author{
Archibaldo Donoso S, María Isabel Behrens P. \\ Clinical variants \\ of Alzheimer disease
}

The heterogeneity and variants of Alzheimer disease (AD) are reviewed. There are cases with a slow or fast evolution and with early or late onset. Most cases are sporadic but there are also hereditary forms. About $50 \%$ of patients show neuropsychiatric disorders (depression and psychoses). Some cases have a greater deficit of right or left hemispheric functions. Among the variants, there are forms that start as pure aphasias, predominantly prefrontal cases and posterior cortical forms. Occasionally AD may simulate other disorders such as supranuclear palsy, corticobasal ganglionar degeneration and JacobCreutzfeldt disease. Finally, there are mixed forms, in which AD is associated with cerebrovascular disease (very commonly) and with other diseases such as dementia with Lewy bodies. We conclude that $\mathrm{AD}$ is a heterogeneous disorder and, therefore, clinical diagnosis may be insufficient. Biological markers and specific imaging studies are needed for a correct clinical diagnosis (Rev Méd Chile 2005; 133: 477-82).

(Key Words: Early onset Alzheimer disease; Focal onset Alzheimer disease; Late onset Alzheimer disease; Psychosis)

Recibido el 21 de julio, 2004. Aceptado el 22 de noviembre, 2004.

Departamento de Neurología y Neurocirugía, Hospital Clínico de la Universidad de Chile. Santiago de Chile.

L a enfermedad de Alzheimer (EA), principal causa de demencia en los adultos mayores, es una enfermedad heterogénea, con manifestaciones variables. Hace cerca de 20 años, Mayeux hizo hincapié en esta heterogeneidad, señalando 4 formas: a) benigna, de lenta progresión; b) mioclónica en sujetos jóvenes y evolución rápida (algunos podrían haber sido enfermedades priónicas); c) extrapiramidal, con síntomas psicóticos y deterioro rápido (algunos podrían ser demencias

Correspondencia a: Archibaldo Donoso S. Fax: (56-2) 7378546. E mail: adonoso@redclinica.uchile.cl. por cuerpos de Lewy); y d) típica1. Recientemente Galton ha destacado esta variabilidad ${ }^{2}$.

El objetivo de esta revisión es mostrar el abanico de manifestaciones de la EA. Para ello revisaremos los siguientes aspectos: a) Curso rápido o lento; b) Formas esporádicas y hereditarias; c) Formas precoces o tardías; d) EA con alteraciones neuropsiquiátricas; e) Formas asimétricas, focales y atípicas; y f) Formas mixtas.

a. Curso rápido o lento. La sobrevida promedio en EA fluctúa entre 7 y 9 años 3,4 . Varios factores influyen en ella. Uno es la severidad de la demencia al consultar, por un curso acelerado, aunque la consulta tardía puede deberse a facto- 
res sociales. Otros indicadores de sobrevida breve son la pérdida de 5 puntos o más del "Minimental test" de Folstein durante el primer año de control, la aparición precoz de signos extrapiramidales 0 frontales, alteraciones de la marcha y caídas $^{4,5}$. También influye la edad de inicio; existen formas preseniles de evolución rápida; pero por razones médicas generales, en un paciente de 65 años se espera una sobrevida de 8,3 años, en otro de 90 sólo de 3,4 años. Según Larson, la mujer sobrevive más que el hombre. La existencia de cardiopatías y diabetes mellitus disminuyen la sobrevida ${ }^{5}$.

b. Formas esporádicas y familiares. La EA es hereditaria en $1 \%$ de los casos. Hay 3 genes cuyas mutaciones pueden expresarse con el fenotipo EA: el gen de la proteína precursora del amiloide (PPA) en el cromosoma 21 (responsable de la asociación entre EA y síndrome de Down), el gen de la presenilina 1 (PS1) en el cromosoma 14 y el de la presenilina 2 (PS2) en el cromosoma $1^{7}$. En estas mutaciones existe una sobreproducción de beta-amiloide, que se encuentra elevado en el plasma $^{8,9}$. Las más frecuentes son las mutaciones de PS1; en 2002, Holmes mencionaba 69 mutaciones del gen de PS1, 12 del gen PPA y 5 de la PS2. Dos años después se conocen más de 100 de la PS1 y por lo menos 6 de la PS2 ${ }^{8}$.

Las mutaciones del gen de PPA, generalmente, causan una EA presenil, entre los 40 y 65 años; explicaría menos del $0,1 \%$ de los casos $^{8}$. Las mutaciones de PS1 y PS2 también se expresan como EA precoz; en las mutaciones de PS1 el curso es rápido, en pocos años ${ }^{7}$. En algunas familias se han descrito mioclonías y en otras paraparesia espástica ${ }^{10,11}$. Las mutaciones son más frecuentes para PS1, menos para la PPA y PS2; en algunos casos la herencia no se debe a ninguna de las mutaciones conocidas ${ }^{12}$.

En la EA tardía (90 ó 95\% de los casos), la historia familiar es un factor de riesgo, probablemente por herencia multifactorial poligénica ${ }^{7}$. En ellas suele encontrarse hasta $20 \%$ de antecedentes familiares de demencia, pero esto se explicaría por la alta incidencia de EA esporádica y otras demencias en adultos mayores ${ }^{3}$.

Muchos genes podrían aumentar el riesgo de EA. El más conocido es el de apolipoproteína E: cuando en vez de los alelos ApoE2 o ApoE3 existen alelos $\mathrm{E} 4$ (que podrían aumentar la agre- gación o disminuir la depuración de la beta amiloide) se favorece la aparición de la EA (esporádica 0 familiar), que puede adelantarse varios años $7,8,13$. En el deterioro cognitivo leve (DCL) aumenta el riesgo de transformación a demencia $^{14}$. Otros genes cuya mutación podría aumentar el riesgo son: de receptores de lipoproteínas de muy bajo peso molecular (VLDL), la butirilcolinesterasa, la enzima convertidora de angiotensina, de la alfa-1 antiquimotripsina, del complejo HLA, de la alfa 2 macroglobulina, de un transportador de serotonina, de la interleukina $1^{7}$.

La degeneración neurofibrilar es importante en la EA, pero no se han encontrado mutaciones de proteína tau como causa de ella. Esto permitiría suponer que en su patogenia es más importante el beta amiloide, o que la degeneración neurofibrilar es un fenómeno más tardío ${ }^{8}$.

En EA con trastornos neuropsiquiátricos también pueden ser importantes los factores genéti$\cos ^{7}$.

c. EA senil y presenil. El límite arbitrario entre las formas seniles y preseniles son los 65 años; el origen de esta distinción tiene sólo razones históricas y ambas son EA. Sin embargo, hay algunas diferencias. Las más importantes clínicamente son las consecuencias familiares. En efecto, si el paciente es añoso y tiene hijos adultos, éstos son capaces de enfrentar la situación; en uno de 50 ó 60 años sus hijos adolescentes serán incapaces de hacerlo.

Las formas hereditarias generalmente son preseniles, y a veces de evolución rápida. Es discutible si el compromiso del lenguaje es más importante en las formas preseniles ${ }^{10,15}$. Algunas formas preseniles hereditarias se asocian a mioclonías, paraparesia espástica, distonía y disartria ${ }^{11}$.

d. Formas neuropsiquiátricas. En algunos pacientes destacan los síntomas neuropsiquiátricos. Cummings ha encontrado apatía (72\%), agitación (60\%), ansiedad (48\%), irritabilidad (42\%), disforia (38\%), conducta motora aberrante (38\%), desinhibición (36\%), delirio lúcido (22\%), alucinaciones (10\%), euforia $(8 \%)^{16}$.

La apatía (falta de motivación, de reacción emocional) puede asociarse a labilidad emocional y egocentrismo. Fue motivo de consulta en $7 \%$ de nuestra muestra ${ }^{3}$. Existe en $30-40 \%$ de los pacientes, 
a veces asociada a depresión. Estos sujetos tienen menor rendimiento cognitivo y en las actividades de la vida diaria; cuando la apatía se asociaba a depresión existía mayor compromiso extrapiramidal ${ }^{17}$.

La depresión puede confundirse con apatía, pero presenta vivencias de tristeza, desesperanza y desvalorización personal, y no sólo pérdida de iniciativa. Se encuentra hasta en $44 \%$ de los pacientes $^{17,18}$. Fue un síntoma inicial en $12,7 \%$ de nuestros $\operatorname{casos}^{3}$ y no se debe a la conciencia de enfermedad. Se ha buscado su sustrato patológico, sin resultados consistentes ${ }^{18,19}$. Se acompaña de quejas, negativismo, inquietud, agresividad, y mayor deterioro cognitivo (que puede mejorar con el tratamiento) ${ }^{19}$. Por otra parte, la depresión sin EA puede asociarse a involución hipocámpica y deterioro cognitivo, y es factor de riesgo de demencia $^{18}$. Las pseudodemencias depresivas, recuperadas con el tratamiento, años después pueden evolucionar hacia una demencia orgánica ${ }^{20}$.

La primera consulta puede ser motivada por agitación o delirios lúcidos ${ }^{3}$. Cummings dice que hay agitación en $60 \%$ de los pacientes, que en $22 \%$ existe un delirio lúcido y en $10 \%$ alucinaciones $^{16,18}$. Los trastornos psicóticos parecen agregarse en dos subtipos: en uno predominan los delirios paranoides (robo, celotipia y otros); en el otro los errores de reconocimiento (de su casa, familiares, de sí mismo en el espejo) y las alucinaciones ${ }^{21,22}$. Las alucinaciones generalmente son visuales. En estos trastornos, los estudios funcionales muestran algunas correlaciones, todavía no concluyentes: hipoperfusión en cingulum anterior cuando hay apatía, parietal posterior derecho cuando hay delirios, hipometabolismo frontal cuando hay depresión, y otras ${ }^{18}$.

Se han descrito correlaciones genéticas para algunos trastornos conductuales. Variaciones en los genes HT2A y HT2C (implicados en esquizofrenia y enfermedad afectiva), y el polimorfismo en genes receptores de dopamina DRD1 y DRD3 se asocian a psicosis y agresividad ${ }^{7}$.

e. Formas asimétricas, focales y atípicas. En las formas asimétricas la involución predomina en un hemisferio; en las focales existe un defecto focal progresivo. También existen variantes frontales y posteriores, y formas atípicas en que la EA simula otras afecciones.
Hay casos en que predominan los defectos visuoespaciales, por mayor compromiso del hemisferio derecho, y casos en que predominan defectos lingüísticos, por mayor compromiso del hemisferio izquierdo. En pacientes con defectos visuoespaciales marcados, Boxer comprobó con resonancia nuclear magnética (RNM) volumétrica una mayor atrofia en el girus temporal inferior derecho, además de la atrofia hipocámpica y cortical generalizada ${ }^{23}$. También existen pacientes con compromiso lingüístico importante o precoz con respecto a los defectos visuoconstructivos. Destacan casos de afasia progresiva en que esta asimetría se exagera y focaliza. En 13 pacientes atípicos con autopsia, Galton encontró 2 afasias progresivas no fluentes, con agramatismo; las lesiones eran perisilvianas anteriores izquierdas, con respeto del hipocampo. Tres casos con lesiones posteriores tenían afasia fluente, con defecto semántico y defectos en la comprensión; y un caso tenía afasia mixta ${ }^{2}$.

En la atrofia cortical posterior destaca el compromiso precoz de las áreas posteriores. En una proporción variable se afectan las áreas o vías occípito parietales, con simultagnosia, ataxia óptica y parálisis psíquica de la mirada, o las áreas occípitotemporales, cuya lesión se expresa como alexia sin agrafia, agnosia visual y prosopagnosia ${ }^{24}$. La mayonía de los casos autopsiados mostraba una EA.

La forma prefrontal podría confundirse con demencias fronto-temporales. En una serie patológica de 63 pacientes con EA, Johnson y cols, encontraron 19 con degeneración neurofibrilar más severa a nivel prefrontal que entorrinal. En 3 existía un claro defecto ejecutivo; también fallaban en memoria y tareas visuoespaciales. La distribución de las placas seniles no difería de la habitual ${ }^{25}$.

La EA puede simular otras afecciones degenerativas. Excepcionalmente, la autopsia de casos clínicamente diagnosticados como parálisis supranuclear progresiva ha mostrado una EA, con cuerpos de Lewy en el tronco cerebral $^{26}$. Varias veces ha simulado una degeneración corticobasal, con distonía asimétrica, mioclonías, etc. El compromiso fronto-parietal asimétrico explicaría los síntomas ${ }^{27}$.

También puede simular una enfermedad de Creutzfeldt-Jakob, con mioclonías, compromiso extrapiramidal y curso subagudo. Incluso el electroencefalograma (EEG) y la proteína 14-3-3 en el 
líquido céfalo-raquídeo (LCR) sugerían una enfermedad por priones ${ }^{28}$. Finalmente, se han descrito casos de EA que se presentaban como epilepsia mioclónica progresiva en adultos jóvenes ${ }^{29}$.

f. Demencias mixtas. La EA puede asociarse a otras afecciones: vasculares, enfermedad de Parkinson, demencia por cuerpos de Lewy difusos (DCL), síndrome de Down y otras. La asociación de EA y lesiones vasculares es muy frecuente y puede influir en la sintomatología. Un paciente con EA puede sufrir una hemorragia o infarto cerebral, con agravación de su cuadro clínico. Por otra parte, en pacientes que tienen EA es frecuente encontrar en tomografía axial computada (TAC) o RNM una hipodensidad periventricular por microangiopatía isquémica $\mathrm{y}$, a veces, infartos lacunares o incluso territoriales. Estos pacientes suelen ser más viejos, con demencia más severa, pero menos patología de $\mathrm{EA}^{30}$. Sin considerar la angiopatía amiloidea, la mitad de los pacientes con EA clínica muestra en la autopsia lesiones vasculares de severidad variable: lagunas, leucoencefalopatía, infartos o hemorragias recientes 0 antiguas ${ }^{30}$.

Los factores de riesgo vascular (hipertensión arterial no controlada, diabetes mellitus, dislipidemias, hiperhomocisteinemia) aumentan el riesgo de demencia ${ }^{31}$. Los pacientes con el alelo ApoE4 tendrían más riesgo de hipercolesterolemia y mayor producción de beta amiloide; el uso de estatinas corrige la dislipidemia y favorecería la vía no amiloidogénica de la PPA ${ }^{32}$. El aumento de beta amiloide en el plasma se ha asociado a lagunas y lesiones isquémicas de sustancia blanca, pero sólo en portadores de ApoE4, que tendría un rol patogénico en el daño vascular 9 .

La angiopatía amiloide (por beta amiloide; existen otras por otros péptidos) se asocia con frecuencia a EA familiar o esporádica ${ }^{33}$. Puede expresarse como hemorragias o infartos córticosubcorticales de pequeño tamaño, a veces recurrentes, que contribuyen a la demencia.

La EA puede asociarse a DCL, que sería la segunda causa de demencia degenerativa ${ }^{34}$. En EA se observan ocasionalmente cuerpos de Lewy; en la DCL es frecuente encontrar lesiones de EA. En 90\% de 98 pacientes con DCL coexistían elementos de EA, y sólo en $10 \%$ se podía hablar de DCL pura ${ }^{35}$. En esta serie, la prevalencia de los signos característicos de la DCL fue baja: $42 \%$ tuvo alucinaciones visuales (más frecuentes cuando había poco componente EA), 55\% compromiso extrapiramidal. Cuando el componente EA era escaso, la mayoría de los casos era diagnosticado como parkinsonismo y se podía plantear el diagnóstico correcto; cuando era importante se diagnosticaba una demencia degenerativa y no $\mathrm{DCL}^{35}$.

La patogenia de la demencia en la enfermedad de Parkinson ha sido muy debatida. Se han señalado 3 alternativas: que la demencia tenga relación con la pérdida de neuronas en la sustancia nigra medial; coexistencia de enfermedad de Parkinson y EA en pacientes añosos; y el compromiso límbico y cortical por cuerpos de Lewy, que sería lo más frecuente ${ }^{34,36}$. En relación con esta última posibilidad, existiría un continuo entre la enfermedad de Parkinson y la DCL.

Finalmente, el síndrome de Down tiene una fuerte asociación con EA. La sobreproducción de la PPA y de beta amiloide hace que todos los sujetos con síndrome de Down tengan la patología de $\mathrm{EA}$ a los 40 años; a los 65, 30 a 75\% presenta una demencia ${ }^{37}$. El riesgo aumenta con el alelo ApoE4, deficiencia estrogénica y aumento del beta amiloide plasmático. Otros investigadores han encontrado que la demencia se adelanta cuando existe un polimorfismo en la región del gen PPA con 3 o más repeticiones de un tetranucleótido en el intrón 7 del gen, y no cuando existe el alelo ApoE4 ${ }^{38}$.

Como casos excepcionales podemos citar la asociación de EA con degeneración corticobasal ${ }^{39}$ y con enfermedad de Creutzfeldt Jakob ${ }^{40}$.

\section{CONCLUSIONES}

La EA es una afección degenerativa, lentamente progresiva, en la cual las imágenes cerebrales habituales son normales o muestran una involución inespecífica. Generalmente, se inicia con trastornos de la memoria episódica y fallas del juicio. La segunda etapa se caracteriza por los defectos del lenguaje, apraxias, síndrome de Gerstmann; el parkinsonismo es tardío y paulatinamente lleva a la tercera etapa final de postración $^{3,4}$. Sobre este esquema básico existen variaciones clínicas. Casi siempre es esporádica, pero existen formas hereditarias; su frecuencia aumenta con la edad, pero hay formas precoces; 
el curso habitualmente es lento, pero puede ser más rápido; pueden agregarse cuadros depresivos y psicóticos. Con respecto a la distribución anatómica de las lesiones, generalmente se inicia en ambos hipocampos, pero puede ser asimétrica 0 predominar en los polos anterior o posterior del cerebro. Además es posible que coexista con otras patologías cerebrales.

Esta variabilidad puede dificultar el diagnóstico y la evaluación del tratamiento. Debemos tener presente que es frecuente que coexista con daño

\section{REFERENCIAS}

1. Mayeux R, Stern $Y$, Spanton S. Heterogeneity in dementia of the Alzheimer type. Neurology 1985; 35: 453-60.

2. Galton CJ, Patterson K, Xuereb JH, Hodgen IR. Atypical and typical presentation of Alzheimer's disease, a clinical, neuropsychological, neuroimaging and pathological study of 13 cases. Brain 2000; 123: 484-98.

3. Donoso A, Quiroz M, Yuls J. Demencia de tipo Alzheimer: experiencia clínica en 71 casos. Rev Méd Chile 1990; 118: 139-45.

4. LieBson E, Albert M. Cognitive changes in dementia of the Alzheimer type. En: Calne DB ed: Neurodegenerative disorders. Philadelphia, WB Saunders Co, 1994.

5. Larson E, Shadien M-F, Wang L, McCormick W, Bowen J, TeRr L ET AL. Survival after initial diagnosis of Alzheimer disease. Ann Intern Med 2004; 140: 501-9.

6. Brookmeyer R, Corrada MM, CuRriero F, Kawas C. Survival following a diagnosis of Alzheimer's disease. Arch Neurol 2002; 59: 1764-7.

7. Holmes C. Genotype and phenotype in Alzheimer's disease. J Psychiatry 2002; 180: 131-4.

8. Selkoe DJ. Alzheimer disease: mechanistic understanding predicts novel therapies. Ann Int Med 2004; 140: 627-38.

9. Van Dijk E, Prins ND, Vermeer S, Hofman A, van Duijn C, Koudstaal P et al. Plasma amyloid beta, apolipoprotein E, lacunar infarcts and white matter lesions. Ann Neurol 2004; 55: 570-5.

10. Kennedy AM, Rossor MN, Hodges JR. Familial and sporadic Alzheimer's disease, en JR Hodges ed: vascular; con menor frecuencia coincide con otras patologías.

En este momento el elemento más útil para el diagnóstico es el juicio clínico, pero en muchas ocasiones se requiere de experiencia con las formas atípicas. Por eso es necesario el desarrollo de marcadores biológicos, el perfeccionamiento de las técnicas de imagen, y si es posible completar el seguimiento de los pacientes hasta el estudio neuropatológico.

Early-onset dementia: a multidisciplinary approach. Oxford University Press, Oxford 2001.

11. Moretti P, Lieberman AP, Widde EA, Giordani BI, KuUin KJ, Koeppre RA et aL. Novel insertional presenilin 1 mutation causing Alzheimer disease with spastic paraparesis. Neurology 2004; 62: 1865-9.

12. Janssen JC, Beck JA, Campbell A, Dickinson NC, Fox RJ, HARNEy RJ ET AL. Early onset familial Alzheimer's disease: mutation frequency in 31 families. Neurology 2003; 60: 235-9.

13. Pastor P, Roe CM, Vimegas A, Bedoya G, ChakraverTY S, GarCía G ET aL. Apolipoprotein E epsilon 4 modifies Alzheimer's disease onset in an E280A PS1 kindred. Ann Neurol 2003; 54: 163-9.

14. Petersen R, Smith GE, Waring SC, Ivnik RJ, TangaLos EG, КокмEN E. Mild cognitive impairment, clinical characterization and outcome. Arch Neurol 1999; 56: 303-8.

15. Seltzar B, SHerwin Y. A comparison of clinical features in early and late onset primary degenerative dementia. One entity or two. Arch Neurol 1983; 40: 143-6.

16. Cummings JL. The neuropsychiatric inventory: assessing psychopathology in dementia patients. Neurology 1997; 48 (supl 6): S10-16.

17. Starkstein S, Petracca G, Chemerinski E, Kremer J. Syndromic validity of apathy in Alzheimer's disease. Am J Psychiatry 2001; 158: 872-7.

18. Assal F, Cummings JL. Neuropsychiatric symptoms in the dementias. Curr Opin Neurol 2002; 15: 44550.

19. Boland RJ. Depression in Alzheimer's disease and other dementias. Curr Psychiat Report 2000; 2: 427-33. 
20. Alexopoulos GS, Meyer BS, Young PC, Mattis S, KAKUME $T$. The course of geriatric depression with "reversible dementia": a controlled study. Am J Psychiat 1993; 150: 1693-9.

21. Cook SE, Miyahara S, Bacanu SA, Pérez-Madrinan O, López OL, Kaufer DI et al. Psychotic symptoms in Alzheimer's disease: evidence for subtypes. Am J Geriatric Psychiatry 2003; 11: 406-13.

22. SulTzER DL Psychosis and antipsychotic medications in Alzheimer's disease, clinical management and research perspective. Dement Geriatr Cogn Disord 2004; 17: 78-90.

23. Boxer AL, Kramer JH, Du AT, Schuff N, Weiner MW, MiLer BL et aL. Focal right inferotemporal atrophy in $\mathrm{AD}$ with disproportionate visual constructive impairment. Neurology 2003; 61: 1485-91.

24. Donoso A, Manterola J. La atrofia cortical posterior, una variante de la enfermedad de Alzheimer. Rev Neurol Arg 2001; 26: 176-80.

25. Johnson JK, Head E, Kim R, StarR A, Cotman C. Clinical and pathological evidence for a frontal variant of Alzheimer's disease. Arch Neurol 1999; 56: 1233-9.

26. Osaki Y, Ben-Shlomo Y, Lees AJ, Daniel SE, Colosimo C, Wenning Q ET AL. Accuracy of clinical diagnosis of progressive supranuclear palsy. Mov Disord 2004; 19: 181-9.

27. Boeve BF, Maraganore DM, Parisi JE, Ahlskog JE, Grafr-Radford N, CASe山i RJ et al. Pathological heterogeneity in clinically diagnosed corticobasal degeneration. Neurology 1999; 53: 795-803.

28. Tschampa HJ, Neumann M, ZerR I, Henkel $K$, Schröter A, Schulz-SchaefFer W et al. Patients with Alzheimer's disease and dementia with Lewy bodies mistaken for Creutzfeldt-Jakob disease. J Neurol Neurosurg Psychiatry 2001; 71: 33-9.

29. Meianson N, Nalbantoglu J, Berkovic S, Melmed C, Anderman E, Roberts LJ et al. Progressive myoclonus epilepsy in young adults with neuropatholo- gical features of Alzheimer' s disease. Neurology 1997; 49: 1732-3.

30. Jewnger KA, MitTer-Ferstl E. The impact of cerebrovascular lesions in Alzheimer disease. J Neurol 2003; 250: 1050-5.

31. Roman GC. Vascular dementia changing the paradigm. Curr Opin Psychiatry 2003; 16: 635-41.

32. Simons M, Keuer P, Dichgans J, Schuiz J. Cholesterol and Alzheimer's disease: is there a link? Neurology 2001; 57: 1089-93.

33. Revesz T, Ghiso J, Lashiey T, Plant G, Rostegno A, Frangione B ET AL. Cerebral amyloid angiopathies: a pathological, biochemical and genetic view. J Neuropath Exp Neurol 2003; 62: 885-98.

34. Galvin JE. Dementia with Lewy bodies. Arch Neurol 2003; 60: 1332-5.

35. Merdes AR, Hansen LA, Jeste DV, Galasko D, HofstetTER CR, Ho GJ ET AL. Influence of Alzheimer pathology on clinical diagnostic accuracy in dementia with Lewy bodies. Neurology 2003; 60: 1586-90.

36. EMRe M. What causes mental dysfunction in Parkinson's disease? Mov Disord 2003; 18: S63S71.

37. Schupf N, Sergievsky GH. Genetic and host factors for dementia in Down' syndrome. Br J Psychiatry 2002; 180: 405-10.

38. Margalo-Lana M, Morris CM, Gibson AM, Tan AL, KAY DW, TYRER SP ET AL. Influence of the amyloid precursor protein locus on dementia in Down syndrome. Neurology 2004; 62: 1996-8.

39. Schneider JA, Watts RL, Georing M, Brewer RP, MIRRA SS. Corticobasal degeneration: neuropathological and clinical heterogeneity. Neurology 1997; 48: 959-69.

40. Tsuchiya K, Yagishita S, Ikeda K, Sano M, Taki K, Hashimoto $K$ et aL. Coexistence of CJD and Alzheimer's disease: an autopsy case showing typical clinical features of CJD. Neuropathology 2004; 24: 46-55. 